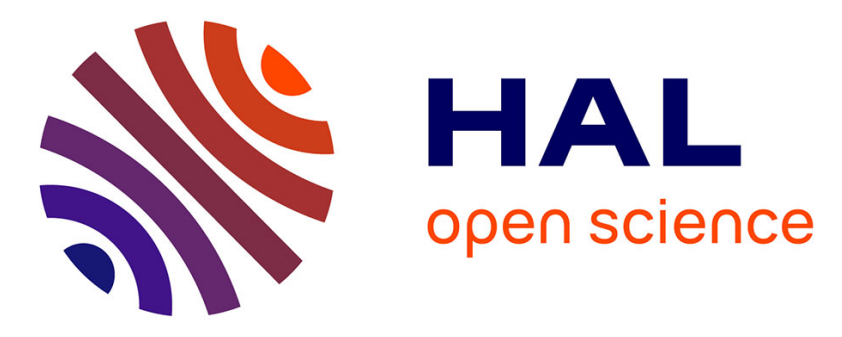

\title{
Antiferromagnetic order and the structural order-disorder transition in the Cd6Ho quasicrystal approximant
}

\author{
A. Kreyssig, Guillaume Beutier, T. Hiroto, M.G. Kim, Gregory S. Tucker, \\ Marc de Boissieu, R. Tamura, A.I. Goldman
}

\section{To cite this version:}

A. Kreyssig, Guillaume Beutier, T. Hiroto, M.G. Kim, Gregory S. Tucker, et al.. Antiferromagnetic order and the structural order-disorder transition in the Cd6Ho quasicrystal approximant. Philosophical Magazine Letters, 2013, 93 (9), pp.512-520. 10.1080/09500839.2013.815375 . hal-00881261

\section{HAL Id: hal-00881261 \\ https://hal.science/hal-00881261}

Submitted on 8 Nov 2013

HAL is a multi-disciplinary open access archive for the deposit and dissemination of scientific research documents, whether they are published or not. The documents may come from teaching and research institutions in France or abroad, or from public or private research centers.
L'archive ouverte pluridisciplinaire HAL, est destinée au dépôt et à la diffusion de documents scientifiques de niveau recherche, publiés ou non, émanant des établissements d'enseignement et de recherche français ou étrangers, des laboratoires publics ou privés. 


\section{Antiferromagnetic order and the structural order-disorder transition in the $\mathrm{Cd}_{6} \mathrm{Ho}$ quasicrystal approximant}

Andreas Kreyssig ${ }^{1}$, Guillaume Beutier ${ }^{2}$, Takanobu Hiroto ${ }^{3}$, Min Gyu Kim ${ }^{1}$, Gregory Tucker $^{1}$, Marc de Boissieu ${ }^{2}$, Ryuji Tamura ${ }^{3}$ and Alan I. Goldman ${ }^{1}$

${ }^{1}$ Ames Laboratory, U. S. DOE and Department of Physics and Astronomy, Iowa State University, Ames, Iowa 50011, USA

${ }^{2}$ SiMAP, Grenoble - UMR 5266 CNRS Grenoble-INP UJF, BP 75, 38402 Saint Martin d'Hères, France

${ }^{3}$ Department of Materials Science and Technology, Tokyo University of Science, Noda, JP-278-8510, Japan 


\title{
Antiferromagnetic order and the structural order-disorder transition in the $\mathrm{Cd}_{6} \mathrm{Ho}$ quasicrystal approximant
}

\author{
We have used x-ray resonant magnetic scattering at the $\mathrm{Ho}_{2}$-edge to elucidate \\ the nature of the antiferromagnetic ordering below $T_{\mathrm{N}}=8.5 \mathrm{~K}$ in $\mathrm{Cd}_{6} \mathrm{Ho}$. The \\ magnetic Bragg peaks are found at the charge forbidden $H+K+L=2 n+1$ \\ positions, referenced to the high-temperature body-centered cubic structure. In \\ general terms, this corresponds to antiferromagnetic arrangements of the Ho \\ moments on adjacent clusters in the unit cell. In contrast to previous studies of \\ $\mathrm{Cd}_{6} \mathrm{Ho}$, we find that there is an abrupt (first-order) transition to a monoclinic \\ structure below $T_{\mathrm{S}} \approx 178 \mathrm{~K}$ and conclude that this may be a general feature of \\ those $\mathrm{Cd}_{6} R(R=$ rare earth $)$ compounds that order magnetically.
}

Keywords: quasicrystal; approximant; antiferromagnetism

\section{Introduction}

Since publication of the discovery of icosahedral quasicrystals by Dan Shechtman in 1984 [1], the nature of the electronic properties of aperiodic systems and, in particular, the intriguing possibility of long-range antiferromagnetic order in quasicrystals, has stimulated a large volume of experimental and theoretical work. To date, however, no "quasi-antiferromagnets" have been discovered and, until very recently, even those periodic approximants to icosahedral quasicrystals that feature local moments (e.g. rareearth ions), exhibit spin-glass-like behaviour at best [2,3]. The recent evidence for long-range antiferromagnetic order in the $\operatorname{Cd}_{6} R(R=$ rare earth $) 1 / 1$ approximants to the Cd-Mg-R quasicrystals offered by magnetic susceptibility and specific heat measurements [4-6], confirmed by scattering experiments [7], has reinvigorated investigations of magnetism in quasicrystals and related periodic approximants. Indeed, the $\mathrm{Cd}_{6} R 1 / 1$ approximants present fascinating structural and magnetic behaviour in addition to the simplicity of binary alloys relative to ternary and higher alloys. 
The room-temperature structure of the $\mathrm{Cd}_{6} R 1 / 1$ approximants may be described as a body-centered cubic (bcc) packing of interpenetrating Tsai-type icosahedral clusters $[8,9]$, which are composed of four successive shells with a Cd tetrahedron at the center. The first shell is a dodecahedron made of $20 \mathrm{Cd}$ atoms, the second shell is an icosahedron of $12 \mathrm{R}$ atoms, the third shell is an icosidodecahedron of $32 \mathrm{Cd}$ atoms and the fourth shell is a rhombic triacontahedron of $60 \mathrm{Cd}$ atoms. For the $1 / 1 \mathrm{Cd}_{6} R$ approximants, the icosahedral clusters placed at the bcc lattice points interpenetrate neighboring clusters along the [111] directions.

For the lighter rare earths $(R=\mathrm{Pr}, \mathrm{Nd}, \mathrm{Sm}, \mathrm{Gd}, \mathrm{Tb}, \mathrm{Dy})$, a structural orderdisorder transition has been reported at $150 \mathrm{~K}<T_{\mathrm{S}}<200 \mathrm{~K}$ such that, below $T_{\mathrm{S}}$, the central Cd tetrahedra order along the [101] directions with alternating orientations [1013]. This results in a doubling and distortion of the unit cell from the high-temperature cubic phase (I $m$-3) to a low-temperature monoclinic phase $(C 2 / c)$. However, for the heavier rare earths $(R=\mathrm{Ho}, \mathrm{Er}, \mathrm{Tm}, \mathrm{Lu})$, no structural transition has been observed [ 6 , 14]. The difference in behaviour between the light and heavy rare earths was explained in terms of steric constraints imposed by the surrounding cages of $\mathrm{Cd}$ and $R$ atoms. The decrease in the lattice constant that follows the lanthanide contraction correspondingly reduces the size of the cavity within the first dodecagonal Cd shell [9] and kinetically limits the reorientation of the $\mathrm{Cd}$ tetrahedra within this cage. This interpretation also seemed consistent with the results of pressure studies on $\mathrm{Cd}_{6} \mathrm{Yb}[15]$.

For many of the $\mathrm{Cd}_{6} R$ alloys, anomalies in the magnetic susceptibility and specific heat measurements have been found that are consistent with long-range magnetic order. For example, relatively sharp transitions appear in the susceptibility and specific heat measurements, for $\mathrm{Cd}_{6} \mathrm{~Tb}$, at approximately $24 \mathrm{~K}$ and $19 \mathrm{~K}[5,6]$. The $24 \mathrm{~K}$ feature has been unambiguously identified as the transition from a paramagnetic 
state to an antiferromagnetically ordered state by x-ray resonant magnetic scattering (XRMS) measurements [7]. The second feature at $19 \mathrm{~K}$ has not yet been identified with a significant change in the magnetic structure and is presently under investigation.

The connection, if any, between the antiferromagnetic order in the $\mathrm{Cd}_{6} R$ series and the higher-temperature structural distortion has not yet been established, although it was speculated that the monoclinic distortion might serve to relieve some degree of magnetic frustration inherent to the nearly perfect icosahedral arrangement of local moments found in the cubic structure [7]. Furthermore, the absence of a structural transition for $\mathrm{Cd}_{6} R$ alloys containing $\mathrm{Ho}, \mathrm{Er}, \mathrm{Tm}$ and $\mathrm{Lu}$ has been associated with a bifurcation of the de Gennes scaling of the Néel temperatures as well as differences in the electronic structure that result in a large residual resistivity at low temperature [6].

The present XRMS and high-energy x-ray diffraction studies of $\mathrm{Cd}_{6} \mathrm{Ho}$ were undertaken to shed further light on this issue. $\mathrm{Cd}_{6} \mathrm{Ho}$ was reported to retain its cubic symmetry to low temperature and order magnetically at $T_{\mathrm{N}}=8.5 \mathrm{~K}[6]$. Our scattering results show that the antiferromagnetic order for $\mathrm{Cd}_{6} \mathrm{Ho}$ manifests the same general features as found for $\mathrm{Cd}_{6} \mathrm{~Tb}$ and there is clear evidence of the same structural orderdisorder transition at $T_{\mathrm{S}}=178 \mathrm{~K}$. This transition is quite sharp in temperature and exhibits hysteresis, indicating a first-order character.

\section{Experimental Details}

Single crystals of $\mathrm{Cd}_{6} \mathrm{Ho}$ were prepared by a self-flux method by melting high purity elements of Cd (99.9999 wt\%) and Ho (99.9 wt\%) in a 9:1 atomic ratio at $993 \mathrm{~K}$ for 24 hours in an alumina crucible sealed inside a quartz tube. This was followed by slow cooling at a rate of $2 \mathrm{~K} /$ hour to $773 \mathrm{~K}$. The remaining Cd melt was then removed by means of a centrifuge, and the alloys were subsequently annealed at $923 \mathrm{~K}$ for 100 
hours to improve the sample homogeneity, followed by further annealing at $473 \mathrm{~K}$ for 3 weeks to reduce point defects. Temperature dependent resistance measurements, as well as electron diffraction patterns taken at high and low temperature, showed no evidence of a structural order-disorder transition down to $20 \mathrm{~K}$.

Since the naturally occurring isotope mixture of $\mathrm{Cd}$ is highly neutron absorbing, magnetic neutron-diffraction measurements are difficult without the appropriate isotopic substitution. Therefore, we have used XRMS to determine the nature of the magnetic order using a millimeter-sized single crystal with well-defined facets normal to the $[1,0,0]$ direction. The XRMS measurement was performed on the 6-ID-B station at the Advanced Photon Source at the Ho $L_{3}$ absorption edge $(E=8.071 \mathrm{keV})$. The incident radiation was linearly polarized perpendicular to the vertical scattering plane ( $\sigma$-polarized) with a beam size of $0.5 \mathrm{~mm}$ (horizontal) by $0.2 \mathrm{~mm}$ (vertical). In this configuration, dipole resonant magnetic scattering rotates the plane of linear polarization into the scattering plane ( $\pi$-polarization). Pyrolytic graphite $[\mathrm{PG}(0,0,6)]$ was used as a polarization analyzer to suppress the charge and fluorescence background relative to the magnetic scattering signal.

In addition to the XRMS measurements, high-energy $\mathrm{x}$-ray $(E=100.2 \mathrm{keV})$ diffraction patterns were recorded in station 6-ID-D at the APS. The use of highenergies minimizes sample absorption, so that the bulk of the sample is probed and large portions of the reciprocal lattice planes are recorded as the sample is rocked through two independent angles about axes perpendicular to the incident beam (see Ref. 16). The direct beam was blocked by a beam stop and diffraction images were recorded by a MAR345 image plate positioned $2862 \mathrm{~mm}$ behind the sample. The beam size was reduced to $0.5 \mathrm{~mm}$ by $0.5 \mathrm{~mm}$ using a slit system. 


\section{Results}

The sample was mounted at the end of the cold finger of a closed-cycle refrigerator with the cubic $(H, K, 0)$ plane coincident with the vertical scattering plane. For the balance of this paper, we will use the cubic notation of the high-temperature phase for the description of the reciprocal space and directions. Above $180 \mathrm{~K}$, only a single Bragg diffraction peak was observed at the charge scattering allowed positions, $H$ $+K+L=2 n,(H, K, L$, and $n$ integer $)$ of the bcc lattice. As the temperature was lowered an abrupt transition occurred resulting in a distribution of peaks about these positions, as illustrated in Figure 1 for the $(10,0,0)$ reciprocal lattice point. Scans along the $[H, 0,0],[0, K, 0]$, and $[0,0, L]$ directions [Figs. 1(a), 1(b) and (c)] revealed at least five distinct domains as shown schematically in Figure 1(f). This distribution of scattering is very similar to what was previously observed for the monoclinic domains at low temperature for $\mathrm{Cd}_{6} \mathrm{~Tb}$ [7], although six rather than five domains were clearly observed in that case. The data in Figure 1, therefore, indicate the presence of a structural order-disorder transition in $\mathrm{Cd}_{6} \mathrm{Ho}$, at $T_{\mathrm{S}}=178 \mathrm{~K}$, that was previously undetected, potentially because the magnitude of the Bragg peak splitting found for $\mathrm{Cd}_{6} \mathrm{Ho}$ is only roughly half of that for $\mathrm{Cd}_{6} \mathrm{Ho}$ with weaker separation of peaks in the diffraction pattern.

The structural order-disorder transition in other $\mathrm{Cd}_{6} R$ alloys is also characterized by the appearance of superlattice peaks, below $T_{S}$, in electron diffraction patterns specified by the propagation vector $(0,1 / 2,1 / 2)$ [10-13]. In Figure 2 we show a split-panel display of the high-energy x-ray diffraction patterns taken with the beam parallel to the $[0,0,1],[1,1,0]$, and $[1,1,1]$ directions of the sample both below (left side) and above (right side) $T_{\mathrm{S}}$. The abovementioned superlattice reflections are clearly evidenced in Figures 2(b) and (c), below $T_{\mathrm{S}}$, confirming the existence of the structural order-disorder 
transition. These superlattice peaks were also observed during the XRMS measurement and the temperature dependence of the intensity of the $(10,1 / 2,1 / 2)$ superlattice reflection is shown in Figure 3. Here, we see that the structural order-disorder transition is quite sharp in temperature and manifests a hysteresis of approximately 1-2 $\mathrm{K}$ on heating and cooling. Therefore, we conclude that this transition is most likely first-order in nature.

Below $T_{\mathrm{N}}=8.5 \mathrm{~K}$, additional magnetic Bragg scattering appeared close to points in reciprocal space corresponding to odd values of the sum $H+K+L=2 n+1(H, K, L$, and $n$ integer) as illustrated in Figs. 1(d) and (e). These figures also show that magnetic Bragg peaks arise from all domains associated with the chemical structure and, as was also found for $\mathrm{Cd}_{6} \mathrm{~Tb}$, the magnetic Bragg peaks are as sharp as the charge Bragg peaks, confirming the long-range character of the magnetic order in $\mathrm{Cd}_{6} \mathrm{Ho}$ below $T_{\mathrm{N}}$. The magnetic transition temperature $\left(T_{\mathrm{N}}=8.5 \mathrm{~K}\right)$ derived from the magnetic susceptibility and specific heat measurements on this sample is in excellent agreement with the onset of magnetic order observed in the XRMS study as shown in Fig. 4. We also see here that all of the monoclinic domains manifest the same evolution of magnetic order as the temperature is lowered below $T_{\mathrm{N}}$.

\section{Discussion and Conclusions}

Inasmuch as the $\mathrm{Cd}_{6} \mathrm{Ho}$ sample studied here clearly manifests a structural order-disorder distortion at a temperature of $T_{\mathrm{S}}=178 \mathrm{~K}$ that is comparable to that in other $\mathrm{Cd}_{6} R$ alloys, we are left with the question of why this was not observed in previous investigations. First, we note that the identification of the structural distortion is generally made by the observation of a kink in the temperature dependence of the resistivity at $T_{S}$, as found for the $\mathrm{Cd}_{6} R$ alloys with $R=\mathrm{Pr}, \mathrm{Nd}, \mathrm{Sm} \mathrm{Gd}$ and $\mathrm{Tb}$ [6], but this feature is much less distinct in $\mathrm{Cd}_{6} \mathrm{Y}$ and $\mathrm{Cd}_{6} \mathrm{Dy}$ and related systems like $\mathrm{Zn}_{6} \mathrm{Sc}[17,18]$, so we must look beyond 
these data. We further note that previous selected area electron diffraction patterns from $\mathrm{Cd}_{6} \mathrm{Ho}$ showed no evidence of the superlattice peaks characteristic of the structural order-disorder transition at low temperatures [14]. However, it has also been established that the physical properties of the $\mathrm{Cd}_{6} R$ and $\mathrm{Zn}_{6} \mathrm{Sc}$ quasicrystal approximants can be quite sensitive to alloy composition, sample preparation protocol and subsequent heat treatments [18]. One possible explanation for the difference observed here is that the sample used in the present study was stored at ambient temperature in an inert atmosphere for approximately one year after synthesis, and this resulted in subtle changes in the structural/chemical order of the sample. In support of this claim, Figure 3(b) displays a measurement of the temperature dependent resistance of the same sample measured in the XRMS experiment which now clearly shows a kink at $T_{\mathrm{S}}$. We note, however, that the Néel temperature for the sample studied in the present investigation $\left(T_{\mathrm{N}}=8.5 \mathrm{~K}\right)$ is the same as that found in previous investigations by other groups, arguing against significant differences in the magnetic properties.

Prior to this investigation, the absence of a structural order-disorder transition in the $\mathrm{Cd}_{6} R$ cubic approximants with $R=\mathrm{Ho}, \mathrm{Er}, \mathrm{Tm}$, and Lu was generally accepted. As described in the Introduction, it was proposed that the decrease in the lattice constant due to the lanthanide contraction reduces the size of the cavity within the first dodecagonal Cd shell below a critical value (at $R=$ Ho) and kinetically limits the reorientation of the $\mathrm{Cd}$ tetrahedral within this cage. Since we have clearly demonstrated that the structural distortion is present in $\mathrm{Cd}_{6} \mathrm{Ho}$, this proposal should be revisited and further structural investigations of the remaining $\mathrm{Cd}_{6} R$ with $R=\mathrm{Er}$, Tm, and $\mathrm{Lu}$ seems necessary.

We have also found that the general features of the antiferromagnetic order in $\mathrm{Cd}_{6} \mathrm{Ho}$ are the same as those found previously for $\mathrm{Cd}_{6} \mathrm{~Tb}$ [7]. With reference to the high 
temperature bcc unit cell description of $\mathrm{Cd}_{6} \mathrm{Ho}$, the antiferromagnetic order breaks the body-centering translational symmetry of the chemical unit cell such that the Ho ions associated with the icosahedral cluster at the corner of the unit cell are antiferromagnetically correlated with the Ho ions associated with the icosahedral cluster at the center of the unit cell. The specific details of the magnetic structure (e.g. moment directions and configurations within a single icosahedral cluster) may, of course, differ between $\mathrm{Cd}_{6} \mathrm{Ho}$ and $\mathrm{Cd}_{6} \mathrm{~Tb}$ (e.g. due to differences in the magnitude of the single-ion anisotropy). A complete specification of magnetic structure will first require a complete description of the chemical structure in the low-temperature monoclinic phase as well as the details of the chemical domain structure in this phase. Further analysis of the magnetic structure can then clarify whether the Ho moments are collinearly aligned or arranged in a more complex manner. 


\section{References}

1. D. Shechtman, I. Blech, D. Gratias, and J.W. Cahn, Phys. Rev. Lett. 53, 1951 (1984).

2. P. Wang, Z. M. Stadnik, K. al Qadi, and J. Przewo'znik, J. Phys.:Condens. Matter 21, 436007 (2009).

3. S. Ibuka, K. Iida, and T. J. Sato, J. Phys.: Condens. Matter 23, 056001 (2011).

4. R. Tamura, Y. Muro, T. Hiroto, K. Nishimoto, and T. Takabatake, Phys. Rev. B 82, 220201(R) (2010).

5. R. Tamura, Y. Muro, T. Hiroto, H. Yaguchi, G. Beutier, and T. Takabatake, Phys. Rev. B 85, 014203 (2012).

6. A. Mori, H. Ota, S. Yoshiuchi, K. Iwakawa, Y. Taga, Y. Hirose, T. Takeuchi, E. Yamamoto, Y. Haga, F. Honda et al. , J. Phys. Soc. Jpn. 81, 024720 (2012).

7. M. G. Kim, G. Beutier, A. Kreyssig, T. Hiroto, T. Yamada, J. W. Kim, M. De Boissieu, R. Tamura and A. I. Goldman, Phys. Rev. B 85, 134442 (2012).

8. C. P. Gomez and S. Lidin, Phys. Rev. B 68, 024203 (2003).

9. S.Y. Piao, C.P. Gómez and S. Lidin, Z. Naturforsch. B 60644 (2006).

10. R. Tamura, K. Edagawa, K. Shibata, K. Nishimoto, S. Takeuchi, K. Saitoh, M. Isobe and Y. Ueda, Phys. Rev. B 72, 174211 (2005).

11. R. Tamura, K. Edagawa, C. Aoki, S. Takeuchi and K. Suzuki, Phys. Rev. B 68, 174105 (2003).

12. R. Tamura, K. Edagawa, C. Aoki, S. Takeuchi and K. Suzuki, J. Alloys Compd. 378, 290 (2004).

13. K. Nishimoto, R. Tamura and S. Takeuchi, Phys. Rev. B 81, 184201 (2010).

14. K. Nishimoto, T. Sato, M. Muraki and R. Tamura, Phil. Mag. 91, 2587 (2011).

15. T. Watanuki, A. Machida, T. Ikeda, K. Aoki, H. Kaneko, T. Shobu, T. J. Sato and A. P. Tsai, Phys. Rev. Lett. 96, 105702 (2006).

16. A. Kreyssig, S. Chang, Y. Janssen, J.-W. Kim, S. Nandi, J. Q. Yan, L. Tan, R. J. McQueeney, P. C. Canfield, and A. I. Goldman, Phys. Rev. B 76, 054421 (2007).

17. J. Hasegawa, R. Tamura and S. Takeuchi, Phys. Rev. B 66, 132201 (2002).

18. T. Ishimasa, Y. Kasano, A. Tachibana, S. Kashimoto and K. Osaka, Phil. Mag. 87, 2887 (2007).

19. R. Tamura, Isr. J. Chem. 51, 1263 (2011). 


\section{Figure Captions}

Figure 1. Scans through the charge peaks at the bcc $(10,0,0)$ diffraction peak position along the (a) $[H, 0,0]$, (b) $[0, K, 0]$, and (c) $[0,0, L]$ directions illustrating the distribution of monoclinic domains below $T_{\mathrm{S}}$. Panels (d) and (e) display the magnetic Bragg peaks, measured at $T=1.6 \mathrm{~K}$, near the $(7,0,0)$ charge-forbidden position. The positions of all measured charge peaks are shown schematically in panel (f).

Figure 2. High-energy x-ray diffraction patterns with the beam parallel to the (a) $[0,0,1]$ direction, (b) $[1,1,0]$ direction, and (c) $[1,1,1]$ direction. The left half of each panel is measured at $T=6 \mathrm{~K}$ well below $T_{\mathrm{S}}$ and the right half at $T=195 \mathrm{~K}$ above $T_{\mathrm{S}}$. Superlattice diffraction spots are visible for positions described by a propagation vector $(1 / 2,1 / 2,0)$ but not in the $(H, K, 0)$ plane in panel (a).

Figure 3. (a) Temperature dependence of the $(10,1 / 2,1 / 2)$ superlattice diffraction peak taken on warming (red symbols) and cooling (blue symbols). The inset shows the temperature region close to the structural order-disorder transition at $T_{\mathrm{S}}=178 \mathrm{~K}$ and shows the hysteresis in the transition. (b) The temperature dependence of the normalized resistivity of the XRMS sample. A kink corresponding to the structural order-disorder transition is found at $T_{\mathrm{S}}$.

Figure 4. (a) Specific heat and (b) magnetic susceptibility measurements on the $\mathrm{Cd}_{6} \mathrm{~Tb}$ sample used for the diffraction measurements. (c) Temperature dependence of the magnetic Bragg peaks from the monoclinic domains close to the bcc $(7,0,0)$ position. 

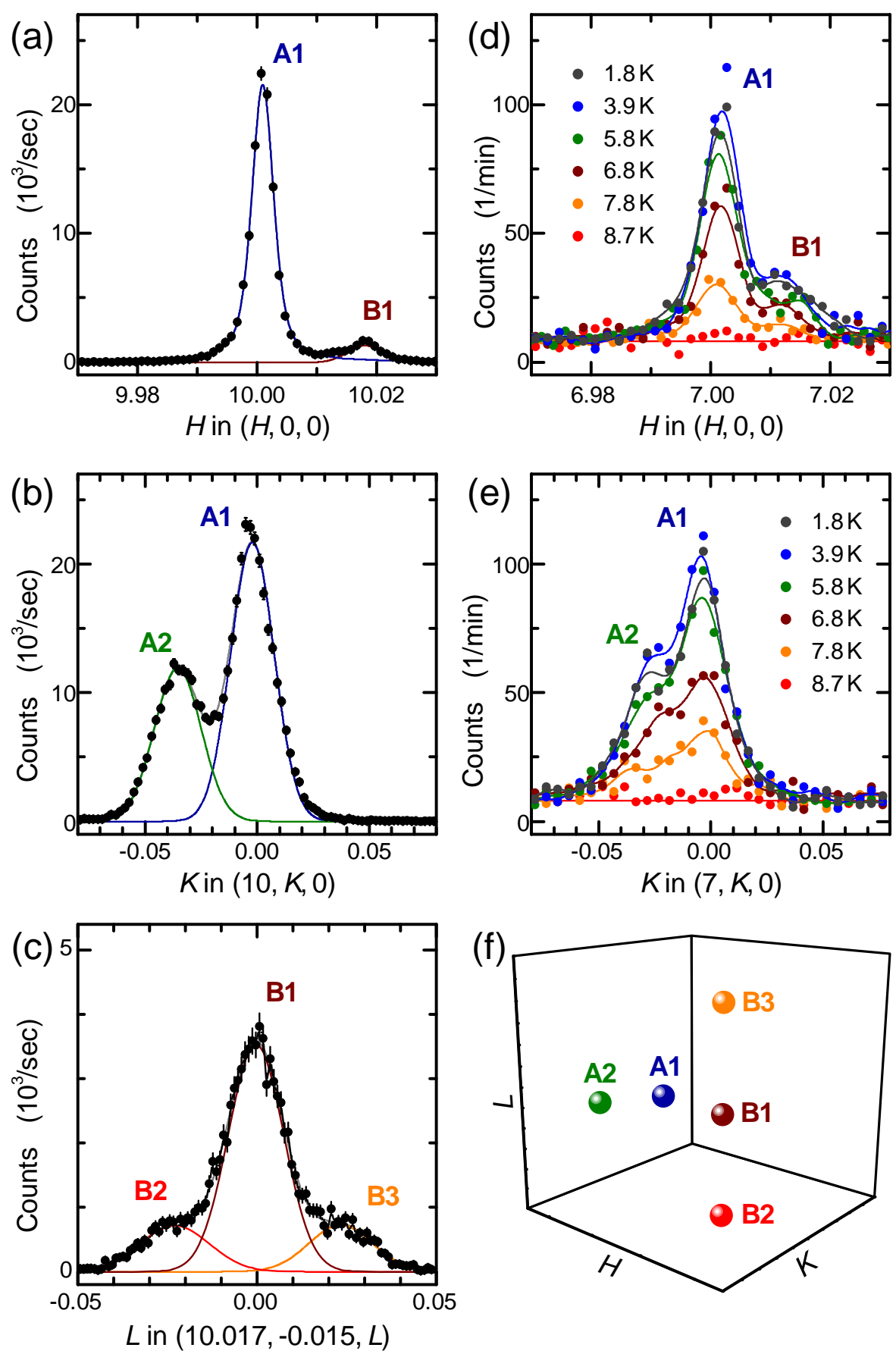

Figure 1 

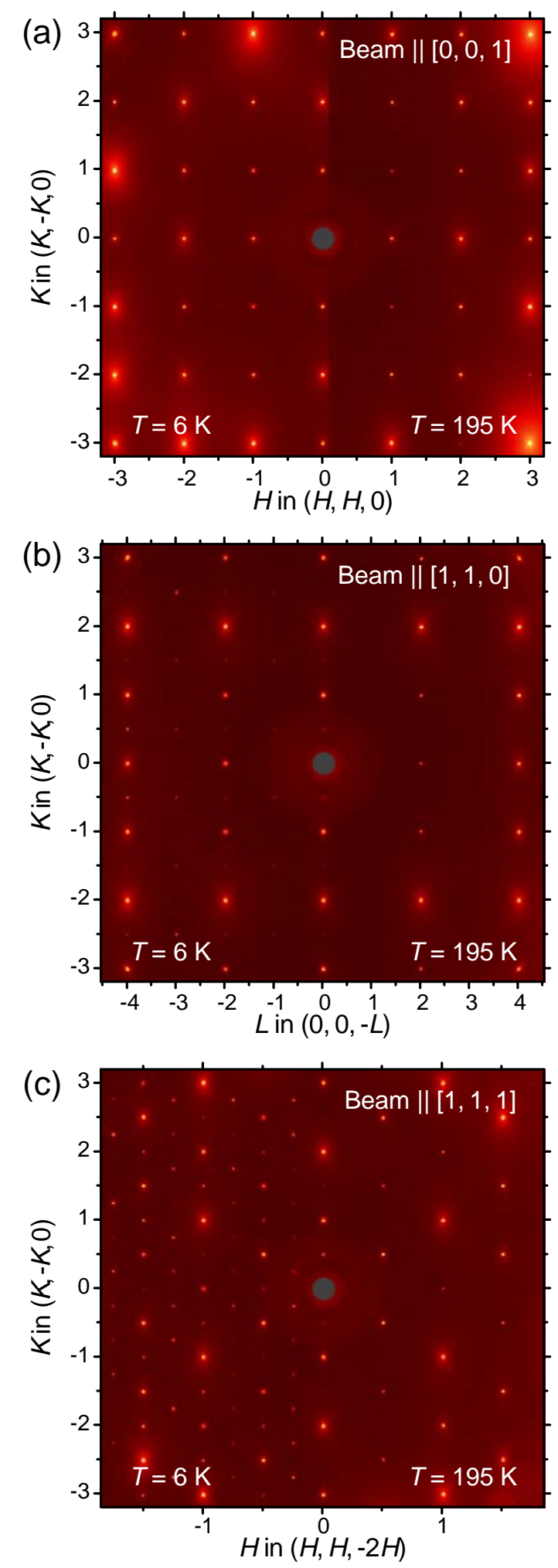

Figure 2 

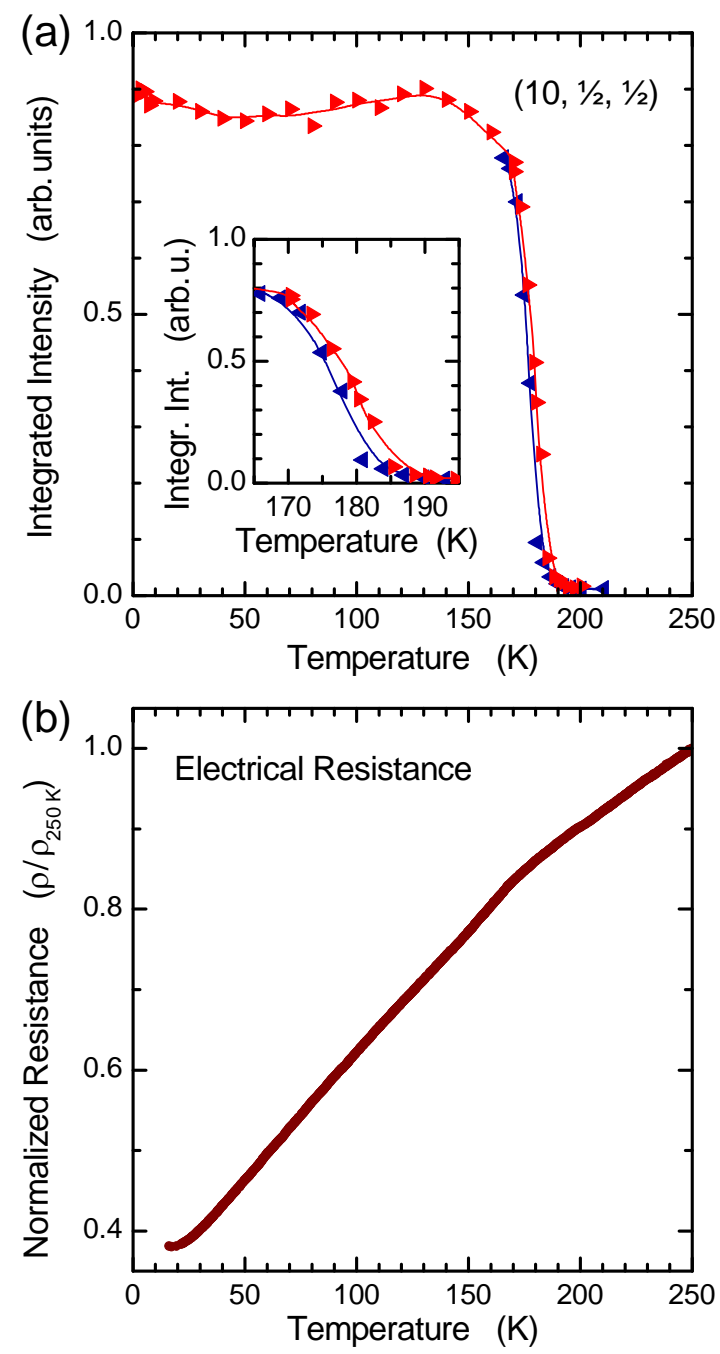

Figure 3 

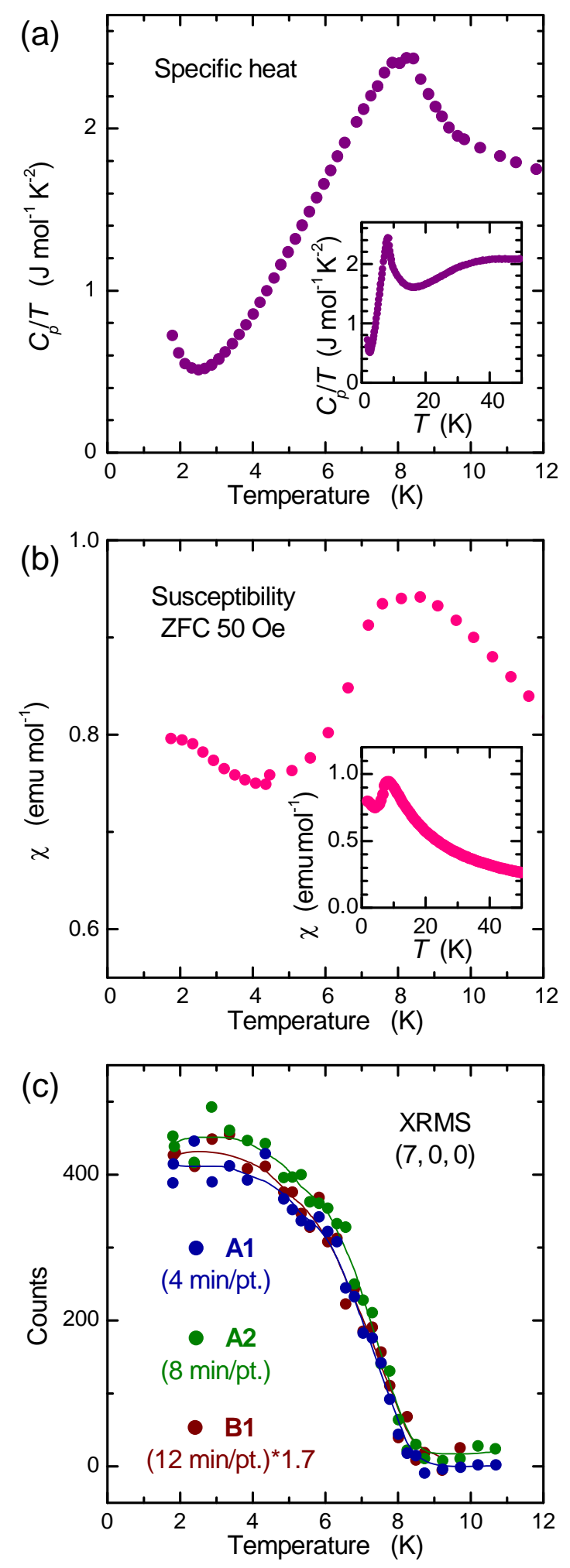

Figure 4 